\title{
Hypergraph Extension of the Alon-Tarsi List Coloring Theorem
}

\author{
Radhika Ramamurthi*, Douglas B. West ${ }^{\dagger}$
}

July, 2002

\begin{abstract}
An Eulerian subgraph in a digraph is a subgraph in which indegree equals outdegree at each vertex. Alon and Tarsi [2] proved that a graph is $d$-choosable when it has an orientation that has no vertex of outdegree at least $d$ and has the property that the numbers of Eulerian subgraphs with even-sized and odd-sized edge sets differ. We generalize this theorem to $k$-uniform hypergraphs, where $k$ is prime. We use a "hypergraph polynomial" and a concept of hypergraph orientation in which a source is chosen from each edge.
\end{abstract}

\section{Introduction}

The notion of list coloring was introduced independently by Vizing [19] and by Erdös, Rubin, and Taylor [10] in the late 1970s. A proper coloring of a graph (or hypergraph) is an assignment of colors to the vertices so that no edge has the same color on all its vertices. In the problem of list coloring, each vertex has an associated list of allowable colors from which its color must be chosen. A (hyper)graph $H$ is $k$-choosable if a proper coloring can be chosen from the lists whenever all the lists have size $k$. The list chromatic number or choice number $\chi_{\ell}(H)$ is the minimum $k$ such that $H$ is $k$-choosable. (Actually, the "lists" are sets, but the term "list" is traditional, and "set coloring" has other meanings.)

\footnotetext{
*Department of Mathematics, California State Univ., San Marcos, CA 92096, rramamu@math.ucsd.edu
}

${ }^{\dagger}$ Department of Mathematics, University of Illinois, Urbana, IL 61801, west@math.uiuc.edu 
Much of the fundamental work on list coloring is surveyed in Alon [5], Tuza [18], and Kratochvil-Tuza-Voigt [13]. We focus on list coloring of hypergraphs. The KratochvilTuza-Voigt paper notes that there are surprisingly few results on list coloring of hypergraphs and that "the structural theory of hypergraph list-coloring" remains largely undeveloped. A few results directly or indirectly discussing list coloring of hypergraphs include KostochkaSteibitz [16], Kostochka-Stiebitz-Wirth [17], and Kahn [14].

In 1992, Alon and Tarsi [2] showed that the choice number of a graph $G$ is at most $d$ when $G$ has an orientation with special structural properties that has no vertex of outdegree at least d. A graph is simply a 2-uniform hypergraph. In this paper, we generalize the Alon-Tarsi Theorem to $k$-uniform hypergraphs when $k$ is prime.

Our proof is algebraic and closely follows the argument of [2]. To motivate our approach, in Section 3 we sketch the main steps in their proof. For the generalization, in Section 4 we define a "hypergraph polynomial" using a $k$ th root of unity $\theta$, generalizing the graph polynomial used in [2]. The polynomial $f_{H}$ is the product of factors for all edges, with edge $v_{i_{0}}, \ldots, v_{i_{k-1}}$ generating the factor $\left(x_{i_{0}}+\theta x_{i_{1}}+\cdots+\theta^{k-1} x_{i_{k-1}}\right)$.

When each $x_{i}$ takes on a value used as a color on the corresponding vertex $v_{i}$, a factor is zero if and only if the corresponding edge is monochromatic, so the value of $f_{H}$ is nonzero if and only if the coloring is proper. The algebraic result used in [2] (stated here in Section 2) implies that $f_{H}$ has value zero for each choice of colors from lists of specified sizes if and only if $f_{H}$ is identically zero.

We use a natural notion of hypergraph orientation to describe the monomials in $f_{H}$; an orientation is a choice of a source vertex from each edge. An orientation in which each vertex $v_{i}$ is chosen $d_{i}$ times as source contributes some power of $\theta$ to the coefficient of $\prod x_{i}^{d_{i}}$.

In Section 5 we establish a bijection between orientations in which each $v_{i}$ is chosen $d_{i}$ times and "balanced" partitions of the edges of one such orientation into $k$ parts. This yields our main result: a sufficient condition for the corresponding coefficient in $f_{H}$ to be nonzero and hence a condition for an upper bound on the choice number of the hypergraph. Section 6 concludes with some examples and questions.

\section{Combinatorial Nullstellensatz}

In this section, we state the algebraic results needed to prove our theorem. For the proofs, we refer the reader to Alon [3]. Applications to the areas of additive number theory, hyperplanes, graphs, and graph colorings are given in [3]. The theorems are special cases of Hilbert's Nullstellensatz (see for example [6, Page 375]); Alon [3] refers to them as the "Combinatorial 
Nullstellensatz".

In a multivariate polynomial, the degree of a nonzero monomial $a x_{1}^{d_{1}} \ldots x_{n}^{d_{n}}$ is the integer $\sum_{i=1}^{n} d_{i}$. If $f$ is a nonzero polynomial expressed as the sum of monomial terms, then the (total) degree of $f$ is the maximum of the degrees of its nonzero monomials. If every nonzero monomial in $f$ has the same degree $t$, then $f$ is homogeneous of degree $t$. The degree of $f$ in $x_{i}$ is the degree of $f$ considered as a polynomial in the one variable $x_{i}$, with the other variables treated as constants.

Let $\mathbb{F}$ be a field. For a polynomial $g \in \mathbb{F}\left[x_{1}, \ldots, x_{n}\right]$ that depends only on $x_{i}$ (that is, the coefficients of monomials involving other variables are zero), it is algebraic convention to write $g\left(x_{i}\right)$. By the "common zeros" of $g_{1}, \ldots, g_{n}$ in this setting, we mean when the arguments are viewed as assigning values to all variables.

Theorem 2.1 (Alon [3]) Let $\mathbb{F}$ be an arbitrary field, and let $f$ be a polynomial in $\mathbb{F}\left[x_{1}, \ldots, x_{n}\right]$. Let $S_{1}, \ldots, S_{n}$ be nonempty subsets of $\mathbb{F}$, and let $g_{i}\left(x_{i}\right)=\prod_{s \in S_{i}}\left(x_{i}-s\right)$. The polynomial $f$ vanishes over all the common zeros of $g_{1}, \ldots g_{n}$ if and only if $f$ lies in the ideal generated by $g_{1}, \ldots, g_{n}$, with $f=\sum_{i=1}^{n} h_{i} g_{i}$ for some polynomials $h_{1}, \ldots, h_{n}$. Moreover, if $f$ and $g_{1}, \ldots, g_{n}$ lie in $R\left[x_{1}, \ldots, x_{n}\right]$ for some subring $R$ of $\mathbb{F}$, then the polynomials $h_{1}, \ldots, h_{n}$ may be chosen in $R\left[x_{1}, \ldots, x_{n}\right]$.

A consequence of this is the following theorem.

Theorem 2.2 (Alon [3]) Let $\mathbb{F}$ be an arbitrary field, and let $f$ be a polynomial in $\mathbb{F}\left[x_{1}, \ldots, x_{n}\right]$. Suppose that $f$ is homogeneous of degree $\sum_{i=1}^{n} t_{i}$, where each $t_{i}$ is a nonnegative integer, and suppose that the coefficient of $\prod_{i=1}^{n} x_{i}^{t_{i}}$ in $f\left(x_{1}, \ldots, x_{n}\right)$ is nonzero. If $S_{1}, \ldots, S_{n}$ are subsets of $\mathbb{F}$ with $\left|S_{i}\right|>t_{i}$, then there are elements $s_{1}, \ldots, s_{n}$ with each $s_{i} \in S_{i}$ such that $f\left(s_{1}, \ldots, s_{n}\right) \neq 0$.

\section{List coloring of graphs}

Assigning identical lists to all vertices shows that $\chi_{\ell}(G) \geq \chi(G)$. Picking colors from lists in an arbitrary vertex order yields $\chi_{\ell}(G) \leq \Delta(G)+1$. No upper bound on $\chi_{\ell}(G)$ in terms of $\chi(G)$ exists; there are bipartite graphs with arbitrarily large choice number. Alon [5] showed that, unlike the chromatic number, the choice number of a simple graph must grow with the average degree: if $G$ has average degree $d$, then $\chi_{\ell}(G) \geq\left(\frac{1}{2}-o(1)\right) \log _{2} d$ [4]. Thus we seek upper bounds in terms of vertex degrees.

The Alon-Tarsi Theorem [2] gives such a bound in terms of a special orientation. When such an orientation exists, the resulting upper bound on $\chi_{l}(G)$ cannot be worse than $\Delta(G)+1$. 
Definition 3.1 For an orientation $D$ of a graph $G$, the outdegree $d_{D}^{+}(v)$ of vertex $v$ is the number of edges with $v$ as source. The indegree is the number of edges with $v$ as sink. An Eulerian subgraph or circulation in a digraph $D$ is a subgraph in which indegree and outdegree are equal at each vertex. The size of a graph or digraph is the number of edges.

The term "Eulerian subgraph" is a bit of a misnomer, because it imposes no connectedness requirement; indeed, the subgraph with no edges is always Eulerian. The term "circulation" is used in network flow theory to mean that inflow equals outflow, so it seems appropriate for this concept.

Theorem 3.2 (Alon-Tarsi [2]) Let $G$ be a graph with an orientation $D$ such that in $D$ the number of circulations with odd size differs from the number of circulations with even size. If each vertex $v$ is given a list of at least $d_{D}^{+}(v)+1$ colors, then $G$ has a proper coloring chosen from the lists.

This theorem is proved in Alon-Tarsi [2]; see also [3, Theorem 7.2]. We briefly describe the proof in the latter paper to show how the results of Section 2 are applied and to motivate our generalization to hypergraphs. The fundamental tool is algebraic, a polynomial associated with the graph.

Definition 3.3 Given a graph $G$ with vertex ordering $v_{1}, \ldots, v_{n}$, let $E^{\prime}(G)=\{(i, j): i<j$ and $\left.v_{i} v_{j} \in E(G)\right\}$. The graph polynomial $f_{G}$ of $G$ is given by $f_{G}\left(x_{1}, \ldots, x_{n}\right)=\prod_{(i, j) \in E^{\prime}(G)}\left(x_{i}-x_{j}\right)$.

Let $v_{1}, \ldots, v_{n}$ be an ordering of the vertices of $G$. A coloring of $V(G)$ can be described as assigning $s_{i}$ to $v_{i}$, where $s_{1}, \ldots, s_{n} \in \mathbb{Z}$. A coloring is proper if and only if the corresponding evaluation $f_{G}\left(s_{1}, \ldots, s_{n}\right)$ of the graph polynomial is nonzero. When we associate a list $S_{i} \subset \mathbb{Z}$ with each vertex of $G$, the existence of a proper coloring of $G$ with each vertex $v_{i}$ colored from its list $S_{i}$ is equivalent to the existence of colors $s_{i} \in S_{i}$ such that $f_{G}\left(s_{1}, \ldots, s_{n}\right) \neq 0$.

Let $D$ be an orientation of $G$ with outdegrees $d_{1}, \ldots, d_{n}$ for $v_{1}, \ldots, v_{n}$, and suppose that $\left|S_{i}\right|=d_{i}+1$ for each $i$. Since the graph polynomial $f_{G}$ is homogeneous of degree $\sum_{i=1}^{n} d_{i}$, Theorem 2.2 applies if the coefficient of $\prod_{i=1}^{n} x_{i}^{d_{i}}$ in $f_{G}$ is nonzero. A sufficient condition for it to be nonzero is obtained by interpreting the coefficient combinatorially in terms of the circulations in $D$. As shown in [2], the absolute value of this coefficient is the difference between the number of circulations of even size and the number of odd size. Hence the bound on choice number follows when $D$ is an orientation satisfying the hypothesis of the theorem.

Alon and Tarsi used the theorem to show that planar bipartite graphs are 3-choosable. Ellingham and Goddyn [11] used it to prove that every $d$-regular, $d$-edge-colorable planar 
multigraph is $d$-edge-choosable. Fleischner and Steibitz [12] applied it to solve a problem of Du, Hsu, and Hwang [8] and a stronger version suggested by Erdös; they proved that every graph on $3 n$ vertices formed by adding $n$ pairwise disjoint triangles to a $3 n$-cycle is 3 -choosable.

\section{The hypergraph polynomial and orientations}

We now consider proper colorings of $k$-uniform hypergraphs. We define a hypergraph polynomial that reduces to the graph polynomial when $k=2$. Like the graph polynomial, this is defined with respect to a fixed ordering of the vertices. We write $\mathcal{E}(H)$ for the edge set of a hypergraph $H$.

Definition 4.1 Let $H$ be a $k$-uniform hypergraph with vertices $v_{1}, \ldots, v_{n}$. For each edge $E=\left\{v_{i_{0}}, v_{i_{1}}, \ldots, v_{i_{k-1}}\right\}$ with $i_{0}<i_{1}<\cdots<i_{k-1}$, let

$$
h(E)=\left(x_{i_{0}}+\theta x_{i_{1}}+\cdots+\theta^{k-1} x_{i_{k-1}}\right),
$$

where $\theta$ is a primitive $k$ th root of unity. The hypergraph polynomial $f_{H}$ is defined by

$$
f_{H}\left(x_{1}, x_{2}, \ldots, x_{n}\right)=\prod_{E \in \mathcal{E}(H)} h(E)
$$

Example 4.2 The edge set of the complete 3-uniform hypergraph on 4 vertices consists of all triples from $\left\{v_{1}, v_{2}, v_{3}, v_{4}\right\}$. The resulting hypergraph polynomial for the vertex ordering $v_{1}, v_{2}, v_{3}, v_{4}$ is $f_{K_{4}^{3}}=\left(x_{1}+\theta x_{2}+\theta^{2} x_{3}\right)\left(x_{1}+\theta x_{2}+\theta^{2} x_{4}\right)\left(x_{1}+\theta x_{3}+\theta^{2} x_{4}\right)\left(x_{2}+\theta x_{3}+\theta^{2} x_{4}\right)$.

Given a $k$-uniform hypergraph $H$, for each $i$ let $L_{i} \subset \mathbb{Z}$ be a list for vertex $v_{i}$. If $H$ has no proper coloring from these lists, then every choice of colors from the lists makes some edge monochromatic. This implies that one of the factors on the right side of (1) is zero.

Conversely, if $f_{H}$ is zero for colors $a_{1}, \ldots, a_{n}$ on the vertices of $H$, then some factor of the form $a_{i_{0}}+\theta a_{i_{1}}+\cdots+\theta^{k-1} a_{i_{k-1}}$ is zero. When $\theta$ is a primitive $k$ th root of unity but $k$ is not prime, this does not imply that $a_{i_{0}}=\cdots=a_{i_{k-1}}$. For example, when $k$ is even and at least 4 , we can give nonzero coefficients to the roots \pm 1 while giving coefficient 0 to the remaining roots. For prime $k$, however, the following holds ([6], Page 405).

Lemma 4.3 Given a prime $k$, let $\theta$ be a primitive $k$ th root of unity. If $a_{0}, \ldots, a_{k-1}$ are rational, then $\sum_{i=0}^{k-1} a_{i} \theta^{i}=0$ if and only if $a_{0}=\cdots=a_{k-1}$.

Henceforth, $H$ denotes a $k$-uniform hypergraph with $k$ prime. We introduce a notion of hypergraph orientation. 
Definition 4.4 Let $H$ be a hypergraph with vertices $v_{1}, \ldots, v_{n}$ in fixed order. An orientation $\vec{H}$ is a choice of a source vertex from each edge in $H$. Letting $d_{i}$ denote the number of edges with $v_{i}$ as source, the source sequence of $\vec{H}$ is $d_{1}, \ldots, d_{n}$. For an edge $\left\{v_{j_{0}}, \ldots v_{j_{k-1}}\right\}$ with $j_{0}<\cdots<j_{k-1}$, the vertex $v_{j_{i}}$ is in position $i$ in the edge. If also $v_{j_{i}}$ is the source in edge $E$, then the weight $w_{\vec{H}}(E)$ is $\theta^{i} x_{j_{i}}$. The weight of $\vec{H}$ is the product $\prod_{E \in \mathcal{E}} w_{\vec{H}}(E)$. The modularity $m(\vec{H})$ is $\left(\sum_{i=0}^{k-1} i m_{i}\right) \bmod k$, where $m_{i}$ is the number of edges with the source in position $i$.

If an oriented hypergraph $\vec{H}$ has source sequence $d_{1}, \ldots, d_{n}$, then the weight and modularity of $\vec{H}$ are related by

$$
w(\vec{H})=\prod_{E \in \mathcal{E}(\mathcal{H})} w_{\vec{H}}(E)=\theta^{m(\vec{H})} x_{1}^{d_{1}} \ldots x_{n}^{d_{n}}
$$

Example 4.5 The Fano plane $F$ is the 3-uniform hypergraph with 7 vertices such that every two edges intersect in exactly one element and every two elements occur together in exactly one edge. If $V(F)=[7]$, then the labels can be permuted so that $\mathcal{E}(F)=$ $\{124,235,346,457,156,267,137\}$.

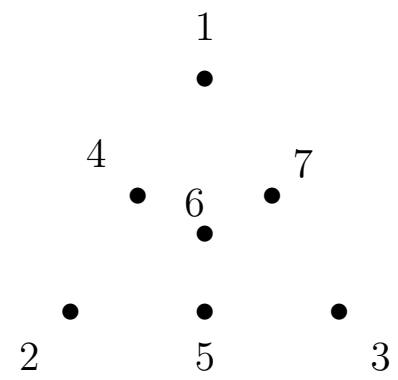

Figure 1

We define an orientation $\vec{R}$ of $F$, indicated by underlining the source in each edge.

$$
\mathcal{E}(\vec{R})=\{\underline{1} 24, \underline{2} 35, \underline{3} 46, \underline{4} 57, \underline{1} 56, \underline{2} 67,1 \underline{3} 7\}
$$

The source sequence of $\vec{R}$ is $2,2,2,1,0,0$. The source in edge 124 is in position 0 , and hence the weight of the edge is $x_{1}$. The weight of $1 \underline{3} 7$ is $\theta x_{3}$, since the source is in position 1 .

The modularity of $\vec{R}$ is $(0 \cdot 6+1 \cdot 1+2 \cdot 0)=1 \bmod 3$. The weight of the orientation is $w(\vec{R})=\left(x_{1}\right)\left(x_{2}\right)\left(x_{3}\right)\left(x_{4}\right)\left(x_{1}\right)\left(x_{2}\right)\left(\theta x_{3}\right)=\theta x_{1}^{2} x_{2}^{2} x_{3}^{2} x_{4}$.

Ignoring the coefficient, the weight of an orientation with source sequence $d_{1}, \ldots, d_{n}$ corresponds to $x_{1}^{d_{1}} \ldots x_{n}^{d_{n}}$, a monomial in $f_{H}$. Conversely, a monomial $x_{1}^{d_{1}} \ldots x_{n}^{d_{n}}$ in $f_{H}$ arises by choosing $v_{i}$ in $d_{i}$ of the edges incident to it (for each $i$ ), and hence it corresponds to an orientation of $H$. Orientations with the same source sequence give rise to the same monomial 
term, but with possibly different coefficients. The coefficient depends on the positions of the sources in the edges. Expanding $f_{H}$ leads to the following expression.

$$
\begin{aligned}
f_{H}\left(x_{1}, x_{2}, \ldots, x_{n}\right) & =\prod_{E \in \mathcal{E}(H)}\left(x_{r_{0}}+\theta x_{r_{1}}+\cdots+\theta^{k-1} x_{r_{k-1}}\right) \\
& =\sum_{\text {all orientations }} w(\vec{H}) \\
& =\sum_{\text {all orientations }} \theta^{m(\vec{H})} x_{1}^{d_{1}} \ldots x_{n}^{d_{n}} \\
& =\sum_{d_{1}+\cdots+d_{n}=e(H)}\left(a_{0}+\theta a_{1}+\cdots+\theta^{k-1} a_{k-1}\right) \prod_{i=1}^{n} x_{i}^{d_{i}},
\end{aligned}
$$

where $a_{i}$ is the number of orientations of $H$ with source sequence $d_{1}, \ldots, d_{n}$ and modularity $i$.

\section{The coefficient of a monomial term}

Consider now a fixed source sequence $d_{1}, \ldots, d_{n}$. Let $\vec{R}$ be an orientation of $H$ with this source sequence. We use " $R$ " to suggest that this is a reference orientation. For any orientation $\vec{H}$ with same source sequence as $\vec{R}$, we partition $\mathcal{E}(\vec{R})$ into classes $B_{0}, \ldots, B_{k-1}$ by comparing sources of edges in $\vec{R}$ and $\vec{H}$.

Let $E$ be an edge of $\vec{R}$ having vertex set $\left\{v_{r_{0}}, \ldots v_{r_{k-1}}\right\}$ with $r_{0}<\cdots<r_{k-1}$ and source $v_{r_{j}}$. When comparing $\vec{R}$ and $\vec{H}$, we put $E$ in $B_{i}$ if the position of the source of $E$ in $\vec{H}$ cyclically follows $v_{r_{j}}$ by $i$ positions. Thus, for a vertex $v$, the number of edges of $\vec{R}$ in $B_{1} \cup \cdots \cup B_{k-1}$ in which $v$ is the source is the number of edges of $H$ such that $v$ is the source in $\vec{R}$ but not in $\vec{H}$.

Also, for each edge in $B_{i}$ where $v$ follows the source by $i$ positions, $v$ is the source in $\vec{H}$ but not in $\vec{R}$. The sum over $i$ of the number of such edges in $B_{i}$ is the number of edges where $v$ is the source in $\vec{H}$ but not in $\vec{R}$.

Since $\vec{R}$ and $\vec{H}$ have the same source sequence, these numbers are equal.

Definition 5.1 Consider an orientation $\vec{R}$ of $\mathcal{H}$. Let $\left(B_{0}, B_{1}, \ldots, B_{k-1}\right)$ be a partition of the edges of $\vec{R}$ into $k$ sets; denote this by $\mathcal{B}$. The modular sum $m(\mathcal{B})$ of a partition $\mathcal{B}$ is defined by $m(\mathcal{B})=\sum_{i=0}^{k-1} i \cdot\left|B_{i}\right| \bmod k$. For each vertex $v$ and each set $B_{j}$ in $\mathcal{B}$, let $d_{B_{j}}^{i}(v)$ denote the number of edges in $B_{j}$ such that $v$ appears $i$ positions after the position of the source of the edge in $\vec{R}$, cyclically. A partition $\mathcal{B}$ is a balanced partition if

$$
\sum_{i=0}^{k-1} d_{B_{i}}^{0}(v)=\sum_{i=0}^{k-1} d_{B_{i}}^{i}(v) \quad \forall v \in \vec{R} .
$$


Note that in $(2)$ the term $d_{B_{0}}^{0}(v)$ cancels from both sides. Therefore, when forming balanced partitions we will mainly consider the edges in $B_{i}$ for $i \neq 0$ and assume that all remaining edges belong to $B_{0}$.

When $k=2$, a balanced partition is a partition of the edges into two sets such that for each vertex $v, d_{B_{1}}^{0}(v)=d_{B_{1}}^{1}(v)$. For a directed graph, $d^{0}(v)$ counts the edges leaving $v$, while $d^{1} v$ counts the edges entering $v$. Thus $B_{1}$ induces a graph in which the indegree of each vertex equals the outdegree; in other words, $B_{1}$ is a circulation in $\vec{R}$.

Example 5.2 Consider again the orientation in Example 4.5:

$$
\mathcal{E}(\vec{R})=\{\underline{1} 24, \underline{2} 35, \underline{3} 46, \underline{4} 57, \underline{15} 6, \underline{2} 67,1 \underline{3} 7\}
$$

Letting $B_{1}=\{\underline{1} 24, \underline{2} 35\}$ and $B_{2}=\{1 \underline{3} 7\}$ yields a balanced partition with modular sum 1 .

To see this, we check equation (2) for each vertex. First $d_{B_{1}}^{0}(1)+d_{B_{2}}^{0}(1)=1$, since 1 is the source once in $B_{1}$ and not in $B_{2}$. Also, $d_{B_{1}}^{1}(1)+d_{B_{2}}^{2}(1)=1$, since 1 follows the source by two positions in the edge in $B_{2}$. Similarly, 2 is the source once in $B_{1}$ and not in $B_{2}$ and follows the source by one position in the edge 124 in $B_{1}$. Vertex 3 is the source once in $B_{2}$ and not in $B_{1}$ and follows the source by one position in the edge 235 in $B_{1}$.

Although 4, 5, and 7 are in edges in $B_{1}$ and $B_{2}$, they are not sources. So, the left side of (2) is zero for each. Since 4 and 5 appear two positions after the source in $B_{1}$, and 7 appears one position after the source in $B_{2}$, the right side of (2) is also zero.

We now reinterpret the coefficient of a monomial in $f$ in terms of balanced partitions of a fixed orientation.

Theorem 5.3 There is a bijection from the set of balanced partitions of $\vec{R}$ to the set of orientations of $H$ with the same source sequence as $\vec{R}$ that maps balanced partitions of $\vec{R}$ with modular sum $j$ to orientations with modularity $j-m(\vec{R}) \bmod \mathrm{k}$.

Proof. The discussion before Definition 5.1 shows how to form a balanced partition of $\vec{R}$ by comparing $\vec{R}$ to $\vec{H}$.

From a balanced partition $\mathcal{B}=\left(B_{0}, B_{1}, \ldots, B_{k-1}\right)$ of $\vec{R}$, we obtain another orientation $\vec{H}_{\mathcal{B}}$ with the same source sequence as follows. Each edge $E$ in $\vec{R}$ lies in some $B_{i}$; we move $i$ positions cyclically after the source of $E$ in $\vec{R}$ to obtain the source of $E$ in $\vec{H}_{\mathcal{B}}$. The number of times $v$ is a source in $\vec{H}_{\mathcal{B}}$ is the total number of times, over all $i$, that $v$ is $i$ positions after the source in $B_{i}$. Since $B_{i}$ is balanced, this equals the number of times $v$ is a source in $\vec{R}$. Hence $\vec{H}_{\mathcal{B}}$ has the same source sequence as $\vec{R}$. This inverts the earlier map.

Also, we obtain $m\left(\vec{H}_{\mathcal{B}}\right)=m(\vec{R})+\sum_{i=0}^{k-1} i \cdot\left|B_{i}\right| \bmod k$. For each edge $E \in B_{i}$, the new weight of $E$ is given by $w_{\vec{H}_{\mathcal{B}}}(E)=\theta^{i} w_{\vec{R}}(E)$, since the source shifts by $i$. Therefore, the number 
of orientations of $H$ with modularity $j$ that have the same source sequence as $\vec{R}$ equals the number of balanced partitions of $\vec{R}$ with modular sum $(j-m(\vec{R})) \bmod \mathrm{k}$.

Recall that we defined $a_{i}$ to be the number of orientations of modularity $i$ with source sequence $d_{1}, \ldots, d_{n}$. By Theorem 5.3, $a_{i}$ equals the number of balanced partitions of $\vec{R}$ with modular sum $(i-m(\vec{R})) \bmod \mathrm{k}$.

Since $f_{\mathcal{H}}\left(x_{1}, \ldots, x_{n}\right)=\sum_{d_{1}+\cdots+d_{n}=e(H)}\left(a_{0}+\cdots+\theta^{k-1} a_{k-1}\right) \prod_{i=1}^{n} x_{i}^{d_{i}}$, this implies that the coefficient of $\prod_{i=1}^{n} x_{i}^{d_{i}}$ is zero if and only if the number of balanced partitions of $\vec{R}$ with modular sum $i$ is independent of $i$.

The following theorem incorporates these combinatorial arguments.

Theorem 5.4 For $k$ prime, let $\vec{R}$ be an orientation of a $k$-uniform hypergraph $H$ such that the number of balanced partitions of $\vec{R}$ with modular sum $i$ is not constant over $i$. If each vertex $v$ is given a list of size at least $d_{\vec{R}}^{0}(v)+1$, then $H$ has a proper coloring from the lists.

Proof. Let $S_{i}$ denote the given lists, and consider the hypergraph polynomial $f_{H}$ defined in 4.1. The polynomial $f_{H}$ is homogeneous of degree $e(H)$. Let the source sequence of the given orientation $\vec{R}$ be $d_{1}, \ldots, d_{n}$. By the remarks following Theorem 5.3, the coefficient of $x_{1}^{d_{1}} \ldots x_{n}^{d_{n}}$ in $f_{H}$ is nonzero. Now Theorem 2.2 applies to guarantee the existence of a proper coloring from the lists.

Example 5.5 Consider again the orientation of $F$ given in Example 5.2. We have shown that $(\{\underline{3} 46, \underline{4} 57, \underline{15} 6, \underline{2} 67\},\{\underline{1} 24, \underline{2} 35\},\{1 \underline{3} 7\})$ is a balanced partition. The only other balanced partition is the trivial one $(\mathcal{E}(\vec{R}), \emptyset, \emptyset)$.

To see this, observe that in the edges $\underline{4} 57, \underline{156}$, and $\underline{2} 67, \underline{1} \underline{7} 7$, one of $5,6,7$ appears immediately after the source (cyclically). These edges cannot be in $B_{1}$ since these vertices are never sources in $\vec{R}$. Similarly, in $\underline{2} 35, \underline{3} 46, \underline{4} 57$, and $\underline{156}, \underline{267}$, one of $5,6,7$ appears two positions after the source cyclically, and these cannot be in $B_{2}$. Further, $\underline{4} 57$ is forbidden from both $B_{1}$ and $B_{2}$, since it is the only edge with 4 as a source. This implies that $\underline{3} 46 \notin B_{1}$ and $\underline{12} \notin \notin B_{2}$. Thus the only edges eligible for $B_{1}$ are $\underline{124}$ and $\underline{2} 35$ and the only edge eligible for $B_{2}$ is $1 \underline{3} 7$. Finally, $\left(\underline{1} 24 \in B_{1}\right) \Rightarrow\left(\underline{2} 35 \in B_{1}\right) \Rightarrow\left(1 \underline{3} 7 \in B_{2}\right) \Rightarrow\left(\underline{1} 24 \in B_{1}\right)$.

Since the total number of balanced partitions is not a multiple of 3 , the number of them having modular sum $i$ cannot be independent of $i$. Thus we conclude that the coefficient of the monomial $x_{1}^{2} x_{2}^{2} x_{3} x_{4}^{2}$ in $f_{F}$ is nonzero, and then Theorem 5.4 implies that $F$ is 3-choosable. The 3-choosability of the Fano plane also follows from a result of Kostochka, Stiebitz, and Wirth [17] extending Brook's theorem to hypergraphs. 


\section{Applications and questions}

It would be interesting to use Theorem 5.4 to obtain the choice number of an infinite family of hypergraphs for which the choice number is not yet known. Unfortunately, counting the balanced partitions of an orientation of a hypergraph is difficult. All that is needed is showing (as in the case of the Fano plane) that some monomial term has a nonzero coefficient. In this section, we describe an infinite family of hypergraphs for which there is only one orientation with a particular source sequence.

We study a family of $k$-uniform hypergraphs with girth $g$ and chromatic number $i$ constructed recursively in Kostochka-Nešetřil [15]. The construction generalizes the classical construction of Blanche Descartes for triangle-free graphs with high chromatic number.

Construction 6.1 Fix the parameters $k$ and $g$. Let $H_{2}$ be a single edge with $k$ vertices. Given $H_{i}$ with $n_{i}$ nodes, construct $H_{i+1}$ as follows. Let $F$ be a $(k-1) n_{i}$-uniform, $(i+1)$ chromatic hypergraph of girth $g$ (this exists by [9]). Let $e_{i}$ be the number of edges in $F$. Take $e_{i}$ copies of $H_{i}$ and adjoin a "central" copy of $F$. Form a 1-1 correspondence between the copies of $H_{i}$ and the edges of $F$. For each copy $C$ of $H_{i}$, divide the vertices of the corresponding edge $e_{C}$ of $F$ into $n_{i}$ sets of size $k-1$ and delete the edge $e_{C}$. Form $n_{i}$ pairwise disjoint new edges of size $k$ by adding each vertex of $C$ to one of the $n_{i}$ pairwise disjoint sets of size $k-1$ in the set $e_{C}$. The resulting hypergraph is $H_{i+1}$.

By induction on $i$, the hypergraph $H_{i}$ has chromatic number at least $i$. To show that $\chi_{\ell}\left(H_{i}\right) \leq i$ using Theorem 5.4, consider an orientation of $H_{i}$ where the vertices of $F$ are never sources. Hence each vertex of each copy of $H_{i}$ must be the source of its edge that uses vertices of $F$. Now delete $F$ and repeat this process on each copy of $H_{i-1}$. Eventually we are left with copies of $\mathrm{H}_{2}$. Since $\mathrm{H}_{2}$ is a single edge, each choice of source yields a unique orientation. Hence there is only one orientation of $H_{i}$ with the source sequence we have specified. This implies that the monomial term corresponding to this source sequence is nonzero.

Since each vertex is chosen as a source at most $i-1$ times in this orientation, the orientation has maximum source degree $i-1$. By Theorem 5.4, we conclude that $H_{i}$ is $i$-choosable.

Kostochka and Nešetřil [15] proved that $H_{i}$ has girth $g$ and chromatic number $k$ and that in every subhypergraph of $H_{i}$ there is a vertex of degree at most $i-1$. This is a more direct way to show that $H_{i}$ is $i$-choosable. Nevertheless, the example shows that our theorem can sometimes be used to obtain the optimal answer for choice number.

Stronger applications may be hard to find. As $k$ increases, hypergraphs become easier to color, but the number of edges and hence source degrees may go up. Also, counting the 
balanced partitions for an orientation is usually difficult. The computation might be easier for sparse hypergraphs, but the choosability of sparse hypergraphs can often be determined by simple probabilistic arguments (using a random coloring).

In the hypergraph case, we have not found an analogue of the easy application of the AlonTarsi Theorem to bipartite graphs. An orientation of a bipartite graph has no circulations of odd size, since such a circulation must have an odd cycle, but bipartite graphs have no odd cycles. On the other hand, the subgraph with no edges is always a circulation of even size. Hence all orientations of a bipartite graph yield nonzero terms in the graph polynomial. it suffices to find an orientation with low outdegrees to obtain a good bound on the choice number. A generalization of this application to $k$-uniform hypergraphs with $k>2$ would require an easily-checked necessary condition for generating nonzero terms in the hypergraph polynomial.

\section{References}

[1] N. Alon and M. B. Nathanson and I. Z. Rusza, The polynomial method and restricted sums of congruence classes, J. Number Theory, 56, 1996, 404-417.

[2] N. Alon and M. Tarsi, Colorings and orientations of graphs, Combinatorica, 12, 1992, $125-134$.

[3] N. Alon, Combinatorial Nullstellensatz, Combin. Probab. Comput., 8, 1999, 7-29.

[4] N. Alon, Degrees and choice numbers, Random Structures Algorithms, 16, 2000, 364-368.

[5] N. Alon, Restricted colorings of graphs, Surveys in Combinatorics, 1993 (Keele), 1993, $1-33$.

[6] M. Artin, Algebra, 1991, Prentice Hall.

[7] B. Bollobás and A. J. Harris, List-colourings of graphs, Graphs and Combinatorics, 1, $1985,115-127$.

[8] D. Z. Du and D. F. Hsu and F. K. Hwang, The Hamiltonian property of consecutive-d digraphs, Math. Comput. Modelling, 17, 1993, 61-63.

[9] P. Erdős and A. Hajnal, On chromatic number of graphs and set-systems, Acta Math. Acad. Sci. Hungar., 17,1966, 61-99. 
[10] P. Erdős and A. L. Rubin and H. Taylor, Choosability in graphs, West Coast Conference on Combinatorics, Graph Theory and Computing, 1979, 125-157.

[11] M. N. Ellingham and L. Goddyn, List edge-colorings of some 1-factorable multigraphs, Combinatorica, 16, 1996, 343-352.

[12] H. Fleischner and M. Stiebitz A solution to a coloring theorem of P. Erdős, Discrete Math., 101, 1992, 39-48.

[13] J. Kratochvíl and Zs. Tuza and M. Voigt, New trends in the theory of graph colorings: choosability and list coloring, Contemporary Trends in Discrete Mathematics (Štiřín Castle, 1997), 1999, 183-197.

[14] J. Kahn, Asymptotically good list-colorings, J. Combin. Theory Ser. A, 73, 1996, 1-59.

[15] A. V. Kostochka and J. Nešetřil, Properties of Descartes' consturction of triangle-free graphs with high chromatic number, Combin. Probab. Comput., 8, 1999, 467-472.

[16] A. V. Kostochka and M. Stiebitz, Colour-critical graphs with few edges, Discrete Math., 191, 1998, 125-137.

[17] A. V. Kostochka and M. Stiebitz and B. Wirth, The colour theorems of Brooks and Gallai extended Discrete Math., 162, 1996, 299-303.

[18] Zs. Tuza, Graph colorings with local constraints - a survey, Discuss. Math. Graph Theory, 17, 1997, 161-228.

[19] V. G. Vizing, Coloring the vertices of a graph in prescribed colors, (in Russian), Diskret. Analiz, 29, 1976, 3-10.

[20] D. B. West, Introduction to Graph Theory, 1996, Prentice Hall. 
Title: Hypergraph Extension of the Alon-Tarsi List Coloring Theorem Abbreviated title: Hypergraph List Coloring

Keywords: list coloring, choosability, hypergraph, orientation

AMS classification: 05C15, 05C65

Authors:

Radhika Ramamurthi

Department of Mathematics

California State University

San Marcos, CA 92096

rramamu@math.ucsd.edu

Douglas B. West

Department of Mathematics

University of Illinois

Urbana, IL 61801

west@math.uiuc.edu

Address for correspondence: Douglas B. West 